\title{
Understanding motivational climates in physical education classes: How students perceive learning and performance-oriented climates by teachers and peers
}

\author{
F. Rodrigues ${ }^{1,2,3}$ (D) D. Monteiro ${ }^{3,4} \cdot$ D. Teixeira ${ }^{5,6} \cdot$ L. $^{\text {Cid }}{ }^{1,3}$ \\ Accepted: 4 September 2020 \\ (C) Springer Science+Business Media, LLC, part of Springer Nature 2020
}

\begin{abstract}
This study aimed to translate and validate the Learning and Performance Oriented in Physical Education Climate Questionnaire (LAPOPECQ) in a sample of Portuguese students. Additionally, this study examined if the measurement would be equivalent across samples and gender. For nomological validity, current study examined the relationships of motivational climates oriented by teachers and peers and behavioral regulations in the physical education context. Data from 536 Portuguese students $($ female $=256)$ aged 10 and 18 years $(M=13.33 ; S D=.47)$ were collected. Participants were involved in regular physical education classes two times per week, according to the Portuguese educational system. The four-correlated factor confirmatory and exploratory model provided acceptable fit to the data. In addition, multigroup analysis showed invariance among groups, namely between calibration and validation samples, and female and male students. Last, significant correlations were found between motivational climates and behavioral regulations as theoretically proposed, confirming nomological validity. The translated LAPOPECQ into Portuguese is a valid instrument assessing students' perception of motivational climates oriented by teachers and peers.
\end{abstract}

Keywords Physical education $\cdot$ Motivational climates $\cdot$ Exploratory factorial analysis $\cdot$ Measurement invariance $\cdot$ Nomological validity

\section{Introduction}

The amount of research in physical education (PE) on enhancing motivation quality is substantial (Vasconcellos et al. 2019). Thus, the construct of perceived achievement has become one of the most popular factors to be measured regarding different adaptive outcomes or maladaptive consequences. Achievement Goal Theory (Nicholls 1984) in classroom

F. Rodrigues

ptfiliperodrigues@gmail.com

1 Sport Science School of Rio Maior (ESDRM - IPSantarém), Av. Dr. Mário Soares $n^{\circ} 110$, Rio Maior, Portugal

2 Life Quality Research Center (CIEQV), Santarém, Portugal

3 Research Center in Sport, Health and Human Development (CIDESD), Vila Real, Portugal

4 Department of Human Kinetics - ESECS, Polytechnique of Leiria, Leiria, Portugal

5 University of Lusófona (ULHT), Lisbon, Portugal

6 Interdisciplinary Center for the Study of Human Performance (CIPER), Lisbon, Portugal settings (Ames and Archer 1988) is defined as the student's view of learning and the goals they choose to pursue in an achievement setting. Based on developmental work with students, scholars concluded that motivational climates are related to the conception of adopted ability acting as goals of action, reflecting the student's personal achievement within a particular context (Papaioannou 1994, 1998).

In the educational context, teachers and peers' behaviors have an important impact on how students are able to regulate their motivation (Vasconcellos et al. 2019) through the motivational climate that they create (Ames 1992). The motivational climate is the contextual situation created by the persons in key positions (e.g., teachers, coaches, trainers) or peers with regard to achievement goal orientations (Duda 2013). Based on the achievement goal framework (Nicholls 1984; Ames 1992), the motivational climate perceived by the student refers to the environment created by the teachers and peers, in class, which is influenced by what they say and do in this context (Duda 2013). In essence, the motivational climates that are created by significant others and perceived by students are assumed to vary in their degree of oriented features. In this sense, two types of climates have been proposed: task-oriented (i.e., learning-oriented) climate that emphasizes learning and personal 
progress, in which task effort is rewarded, and mistakes are a part of the learning process; and ego-oriented (i.e., performance-oriented) climate that emphasizes comparison among students, the demonstration of competence when compared to others, and in which the end results are rewarded and mistakes are punished (Standage et al. 2007).

Achievement goals have been linked in previous literature with other contemporary motivational theories. In fact, variations in achievement and success have been associated with different types of motivation (Duda and Ntoumanis 2003). As proposed by Ryan and Deci (2017), contextual factors such as motivational climates oriented by others will promote selfdetermined types of motivation, hence, the motivational framework used by researchers to determine the quality of motivation is Self-Determination Theory (Ryan and Deci 2019). This motivational theory considers behavioral regulations as "engines" of human motivation. Thus, cognitive, emotional, and behavioral outcomes are dependent on the degree of self-determined regulation. This theoretical framework of human motivation proposes that motivation can take distinct forms differing from one another in function of their degree of self-determined behavior (Ryan and Deci 2017). Autonomous motivation is experienced when individuals engage in behaviors for reasons that are perceived as volitional. In opposition, controlled motivation is experienced when individuals engage in behaviours for reasons that are perceived as internal or external pressures (Ryan and Deci 2017).

\section{Motivational Climates and Behavioral Regulation in the Physical Education Setting}

Based on previous literature (Bortoli et al. 2017; Cid et al. 2019; Ntoumanis and Biddle 1999), learning-oriented and oriented climates are associated with positive outcomes such as increased levels of enjoyment, autonomous motivation, pleasant emotional experiences, and intentions towards leisure-time sport participation. On the other hand, performance-oriented and oriented climates, which are tied to students' perceptions of greater differential treatment and normative references are related to maladaptive achievement outcomes (Duda and Ntoumanis 2003). These results have been recently corroborated in a meta-analysis conducted by Vasconcellos et al. (2019), showing learning-oriented motivational climate to be positively associated with autonomous motivation and performance-oriented climate to be positively associated with controlled motivation in the PE context.

\section{Assessing Motivational Climates and Existing Limitations}

Looking at previous studies, Papaioannou (1994) was the first to attempt to measure perceptions of motivational climates exhibited by teacher and peers at the same time in PE classes, using his own created Learning and Performance Oriented in Physical Education Climate Questionnaire (LAPOPECQ). This measure comprised learning and performance-oriented climates by peers and the learning-oriented motivational climate by teachers. Papaioannou (1998) followed up his previous work by inserting a new factor assessing performanceoriented climates by teachers turning the assessment of motivational climates oriented by peers and teachers complete within the achievement goal framework.

This questionnaire has been applied in several countries namely in Spain (Cervelló-Gimeno et al. 2010), USA (Flores et al. 2008), UK (Warburton 2017) and in Portugal (Cid et al. 2019). However, concerning the application of the LAPOPECQ in Portuguese students (Cid et al. 2019), the authors have used the measure without proper validation. As stated by several authors (Ntoumanis and Biddle 1999; Ryan 1995), research should be performed within specific cultures and scales validated in one context should not be used in another prior to appropriate validation. As a matter of fact, Chen (2008) has been arguing the use of validated scales before conducting complex analysis, since the lack of construct validity could bias reported results. Hence, to the best of our knowledge, no research has ever analyzed and validated the LAPOPECQ measuring both motivational climates oriented by PE teachers and peers in a sample of Portuguese students.

In agreement with past literature (Cervelló-Gimeno et al. 2010; Cid et al. 2019; Papaioannou 1998), the examination of learning and performance climates and the way they are related to behavioral regulations proves to be crucial. Higher levels of autonomous motivation has shown to predict positive outcomes related to academic success (Bortoli et al. 2017; Cid et al. 2019; Huéscar Hernández et al. 2020), thus, how students perceive PE teachers and peers' learning and performance climates should be assessed as a way to understand academic performance (Warburton 2017). In addition, it is essential to measure invariance between groups in order to assess empirically the universality of learning and performance dimensions defined by the achievement goal theory across groups. Thus, the application of the LAPOPECQ for professional and scholar purpose should only be conducted after robust assessment of the factor structure in a sample of students with different characteristics.

\section{Current Research}

As stated by previous studies (Papaioannou 1994, 1998), more studies are needed to extend the validity of the LAPOECQ as a way to confirm its validity in cultures and languages different from the original (i.e., Greece). Cid et al. (2019) have stated as limitation that they used the LAPOPECQ without proper psychometric testing, suggesting 
the need to examine the factor structure of this scale in forthcoming studies. In fact, the lack of psychometric testing of measures may result in misleading results in their presumed generalizability as they are crucial in psychological theories (Chen 2007) such as achievement goal theory and selfdetermination theory. Thus, this study aimed to translate and validate the LAPOPECQ in a sample of Portuguese students. This study was threefold, namely: first, we translated and validated the scale into Portuguese students; second, measurement invariance was carried out between two samples of Portuguese students (i.e., calibration and validation samples) and gender; and, last, this study intends to examine nomological validity using bivariate correlation data from behavioral regulations (i.e., autonomous and controlled motivation).

\section{Methods}

\section{Participants and Procedures}

Data from 536 Portuguese students (female $=256$ ) aged 11 to 17 years $(M=13.33 ; S D=.47)$ were collected. Participants were enrolled in regular physical education classes, two times per week, according to the Portuguese educational system. The calibration sample was composed by 254 participants (female $=132)$ aged 10 to 17 years $(M=12.99 ; S D=.32)$; and the validation sample encompassed 282 students (female $=124)$ aged 10 to 18 years $(M=13.55 ; S D=.42)$.

Procedures were in accordance with the Helsinki declaration and its later amendments. Approval was obtained by the ethical committee prior to data collection. Then, school boards were contacted, research objectives explained, and agreement was attained. PE teachers were reached and data collection procedures were described. Students aged equal or above 18 years and parents or legal guardians of underaged participants were asked to participate voluntarily in this study, signing informed consent. Afterwards, students completed a multisection survey in a classroom setting without the presence of the PE teacher. This sample was designated as the calibration sample of this study. Six months after, we collected data from a new sample (i.e., validation sample). Time taken to complete the survey was approximately $15 \mathrm{~min}$. No counterpart was given; however, students were thanked for their contribution.

\section{Instruments}

Students completed the Portuguese translated Learning and Performance-Orientation in Physical Education Classes Questionnaire (LAPOPECQ; Papaioannou 1994, 1998). The peers' learning and performance-oriented climates and the learning-oriented climate by PE teacher factors were retrieved from the LAPOPECQ (Papaioannou 1994). The performance- oriented climate by the teacher factor was considered from the LAPOPECQ validated by Papaioannou (1998). This scale comprises 22 -items split into four factors, namely: learningoriented climate by peers ( 5 items; item example “... Students feel good when others learn something new"), performanceoriented climate by peers ( 5 items; item example "... Students try to gain rewards by outperforming others"), learningoriented climate by the PE teacher (6 items: item example "... The teacher looks most satisfied when every student learns something new"), and performance-oriented climate by the PE teacher (6 items: item example "... The teacher looks completely satisfied with those students who manage to win with little effort"). All items are followed by the sentence "During physical education..." and participants are asked to respond to each item using a 5-point scale ranging from 1 ("totally disagree") and 5 ("totally agree").

Regarding translation procedures, we followed Brislin $(1970,1980)$ recommendations, specifically: a) preliminary translation was conducted by four experts with higher education in English-Portuguese language, in which researchers designed the first version of the scale; $b$ ) afterwards, four specialists in different scientific expertise (English-Portuguese teacher, physical education teacher, sport psychologist, and researcher in sports science) reviewed the first version of the scale generating the second version of the scale by incorporating their suggested modifications; c) the second version of the scale was reviewed by four other specialists (physical education teacher, psychologist, student psychologist, and researcher in sports science), who, together with the first board of specialists came to a consensual judgement of the content of the new version. In this stage, items had already been translated and adapted to Portuguese; d) the third version of the scale was administered to 40 bilingual college students in the field of sports science with experience for testing English/ Portuguese questionnaires. According to their suggestions, small adjustments were made resulting in a fourth version; and, e) final revisions in which two Portuguese teachers reviewed the fourth version (i.e., syntax, spelling, and grammar) were made resulting in the final Portuguese version of the LAPOPECQ.

Behavioral regulations were measured using the Perceived Locus of Causality Questionnaire Portuguese version (PLCQP; Fernandes and Vasconcelos-Raposo 2005). This scale comprises twenty items assessing amotivation (5 items; example "I do not know why I do physical education classes"), external (5 items; example "I do physical education classes because It is mandatory"), introjected (5 items; example "I do physical education classes because I want to be perceived as a good student"), identified regulation (5 items; example "I do physical education classes because I want to learn new skills"), and intrinsic motivation (5 items; example "I do physical education classes because it is fun"). Participants responded to each statement using a 5-point scale anchored 
from 1 ("totally disagree") to 7 ("totally agree"). In this study, means for controlled motivation (i.e., external and introjected regulation) and autonomous motivation (i.e., identified regulation and intrinsic motivation) were calculated considering previous assumptions (Cid et al. 2019).

\section{Statistical Analysis}

Data was screened for missing values and participants with more than $5 \%$ of absent data were removed from further analysis. For questionnaires with less than $5 \%$ of missing data, missing entries were imputed using multiple imputation procedures (Allison 2000). Descriptive statistics, composite reliability, and correlation coefficients were calculated for each item using IBM SPSS STATISTICS v.23. Cutoffs for normality were considered based on existing guidelines (Gravetter and Wallnau 2014) considering as acceptable scores within range for skewness $(-2 /+2)$ and kurtosis $(-7 /+7)$. For composite reliability, Raykov formula was used considering coefficients equal or above 0.70 as satisfactory (Raykov 1997).

\section{Factor Structure}

All analyses were performed using Mplus 7.4 (Muthén and Muthén 2010) and estimates with the Maximum Likelihood Robust estimator, since it provides standard errors and tests of model fit that are robust to non-normality of the data. Confirmatory Factor Analysis (CFA) and Exploratory Structural Equation Modeling (ESEM) model specifications were performed according to several recommendations (Marsh et al. 2019; Morin et al. 2015) to analyze the factor structure of the LAPOPECQ. Specifically: a) one factor model (Model 1) considering a global motivational climate factor; $b$ ) two-correlated factor CFA (Model 2) and ESEM (Model 3) models (learning and performance climates); and, c) fourcorrelated factor CFA (Model 4) and ESEM (Model 5) models (learning and performance-oriented climates by peers, as well as learning and performance-oriented climates by PE teachers).

In the CFA model specifications, items were only allowed to estimate a priori factors, factors were allowed to correlated, and no cross-loadings were estimated. In the ESEM models, the factors were defined as in the CFA models, however, cross-loadings were freely estimated but forced to a target value of zero using target oblique rotation procedure (Browne 2001).

Since the chi-square $\left(\chi^{2}\right)$ test of exact fit is oversensitive to sample size and model complexity, model fit was examined and relied on the following absolute and incremental indexes: Comparative Fit Index (CFI); Tucker-Lewis Index (TLI); Standardized Root Mean Square Residual (SRMR); Root Mean Square Error of Approximation (RMSEA) and its Confidence Interval at 90\% (CI90\%). According to several authors (Byrne 2011; Hair et al. 2019; Marsh et al. 2019), values greater .90 for CFI and TLI are considered to indicate acceptable fit, whereas values smaller than .80 for SRMR and RMSEA support adequate model fit. Convergent validity was assessed according to the Average Variance Extracted (AVE), accepting scores above 0.50 (Hair et al. 2019). Discriminant validity was achieved if squared correlation scores were below AVE (Byrne 2011).

\section{Multigroup Analysis}

Multigroup analysis was conducted in order to assess invariance between groups with different characteristics (Byrne 2011). For this study, samples (i.e., calibration and validation) and gender (i.e., female and male) were considered for multigroup analysis of the LAPOPECQ. Recommendations from several authors were followed (Byrne 2011; Sass 2011; Cheung and Rensvold 2002) for conducting multigroup analysis, namely: a) the measurement model should fit in each group independently; and, b) differences in CFI $(\Delta \mathrm{CFI})$ and TLI ( $\Delta$ TLI) should be $\leq 0.01$ between configural and other models, namely metric, scalar, and residual (Chen 2007; Cheung and Rensvold 2002; Marsh et al. 2004).

\section{Nomological Validity}

Nomological validity, referred as the degree to which predictions in a formal theoretical framework containing constructs of interest are confirmed, was evaluated considering Pearson correlations between the motivational climates inherent from achievement goal theory and behavioral regulations based on self-determination theory. Thus, LAPOPECQ factors and autonomous and controlled motivation were correlated to examine nomological validity setting significance level at $p<.05$ and $p<.01$ as significant.

\section{Results}

\section{Preliminary Analysis}

Missing values were below 5\%, thus, missing data were imputed using multiple imputation procedures. Descriptive statistics, AVE, and correlations are displayed in Table 1. Higher means in both learning-oriented climates as well as on autonomous motivation were displayed compared to performanceoriented climates and controlled motivation. Skewness and kurtosis were contained within cutoffs in all variables suggesting normal distribution. Convergent and discriminant validity were examined using the four-correlated factor CFA model. Convergent validity was achieved since AVE scores were above cutoffs as seen in Table 1. Discriminant validity was also achieved since squared correlations among constructs were below AVE scores. 
Table 1 Descriptive statistics, average variance extracted, and correlations

\begin{tabular}{|c|c|c|c|c|c|c|c|c|c|c|c|}
\hline & \multirow[t]{2}{*}{ M } & \multirow[t]{2}{*}{ SD } & \multirow[t]{2}{*}{$\mathrm{S}$} & \multirow[t]{2}{*}{$\mathrm{K}$} & \multirow[t]{2}{*}{ AVE } & \multicolumn{6}{|c|}{ Correlations } \\
\hline & & & & & & 1 & 2 & 3 & 4 & 5 & 6 \\
\hline 1. Learning-oriented climate by PE Teacher & 4.15 & .54 & -.51 & .38 & .53 & 1 & .01 & .37 & .03 & .04 & .36 \\
\hline 2. Performance-oriented climate by PE Teacher & 2.83 & .71 & -.04 & .05 & .61 & $.10^{*}$ & 1 & .07 & .07 & .00 & .04 \\
\hline 3. Learning-oriented climate by Peers & 4.09 & .57 & -.41 & -.02 & .61 & $61 * *$ & $.26^{* *}$ & 1 & .12 & .10 & .38 \\
\hline 4. Performance-oriented climate by Peers & 3.04 & .80 & -.29 & -.01 & .59 & $-.17 * *$ & $.27 * *$ & $-.35 * *$ & 1 & .22 & .02 \\
\hline 5. Autonomous Motivation & 5.66 & 1.00 & -.74 & .53 & .72 & $.21 * *$ & .05 & $.31 * *$ & $.47 * *$ & 1 & .12 \\
\hline 6. Controlled Motivation & 3.29 & 1.29 & .33 & -.38 & .62 & $-.60 * *$ & $.20 * *$ & $-.62 * *$ & $.14 * *$ & $-.34 * *$ & 1 \\
\hline
\end{tabular}

Note: $\mathrm{M}$ = Mean; $\mathrm{SD}=$ Standard Deviation; $\mathrm{S}$ = Skewness; $\mathrm{K}=$ Kurtosis; $\mathrm{AVE}=$ Average Variance Extracted; below diagonal line = correlations; above diagonal line $=$ squared correlations; $* p<0.05 ; * * p<0.01$

\section{Factor Structure}

The one factor model (Model 1) as well as the two-correlated factor CFA (Model 2) and ESEM (Model 3) model specifications did not achieve acceptable fit as seen in Table 2. However, both the four-correlated factor CFA (Model 4) and ESEM (Model 5) models provided acceptable fit to the data as seen in Table 2.

Regarding factor loadings, items loaded their pre-defined factor significantly in the four-factor CFA specification. Similar trends were also observed in the four-correlated factor ESEM model. Only item 5, item 12, and item 22 in the fourfactor CFA and several items (i.e., 5, 7, 11, 19, 20, 21, 22) in the four-factor ESEM model presented factor loadings below recommended. However, their removal did not increase model fit. Additionally, items loaded significantly their respective factor and composite reliability coefficients were above the 0.70 cutoff value (as seen in Table 3 ) in both four-correlated factor CFA and ESEM models considering 22 items. Therefore, items were maintained for further analysis.

\section{Multigroup Analysis}

For measurement invariance analysis, we used the fourcorrelated factor CFA model. The measurement model fit in each group (see Table 2) respecting the first step of multigroup analysis. Invariance assumptions were respected between configural model and other models (i.e., weak factorial, strong, and strict factorial) since differences in CFI and TLI were below cutoffs. Thus, the factor structure withholds construct equivalence between samples and between gender. For more details see Table 4 on the differences in CFI and TLI.

\section{Nomological Validity}

Correlations are significant as theoretically expected: i) learning-oriented climates are positively correlated with autonomous motivation and negatively associated with controlled motivation; ii) performance-oriented climates are positively correlated to controlled motivation negatively associated with autonomous motivation. Performanceoriented climates by peers was positively correlated with autonomous motivation $(\beta=0.47, p<0.01)$. Contrarily, learning-oriented climates by peers was negatively correlated with controlled motivation $(\beta=-0.62, \mathrm{p}<0.01)$ as seen in Table 1.

\section{Discussion}

This study aimed to translate and validate the LAPOPECQ in a sample of Portuguese students in the physical education context. In addition, we examined the equivalence of the scale between two samples of Portuguese students and between gender. Last, we examined nomological validity of the motivational climate factors with autonomous and controlled motivation. Our results suggest that the LAPOPECQ (Papaioannou 1994, 1998) for the four-correlated factor model specifications measuring learning and performance-oriented climates by PE teachers and peers according to the achievement goal framework did fit well in a sample of Portuguese students. Significant relationships between these constructs were found as theoretically expected (Papaioannou 1998; Pineda-Espejel et al. 2017).

\section{Factor Structure}

Initially, we developed the Portuguese version of the LAPOPECQ and analyzed its dimensionality and validity testing several CFA and ESEM model specifications. The four-correlated factor CFA and ESEM model on the 22-item scale provided acceptable fit to the data in the calibration and validation sample. Specifically, the model extracted fourcorrelated dimensions assessing learning and performanceoriented climates by PE teachers and peers. These results support the original LAPOPECQ (Papaioannou 1994, 1998) as 
Table 2 Model fit indexes

\begin{tabular}{|c|c|c|c|c|c|c|}
\hline Model & $\chi^{2}$ & $\mathrm{df}$ & CFI & TLI & SRMR & RMSEA $[90 \% \mathrm{CI}]$ \\
\hline 1. One factor model & $2016.345^{*}$ & 209 & .345 & .276 & .124 & $.115[.110, .119]$ \\
\hline 2. Two-correlated factor CFA & $1197.744 *$ & 208 & .641 & .602 & .082 & $.085[.081, .090]$ \\
\hline 3. Two-correlated factor ESEM & $1414.241 *$ & 188 & .747 & .689 & .059 & $.073[.069, .076]$ \\
\hline 4. Four-correlated factor CFA & $902.181 *$ & 203 & .917 & .900 & .048 & $.049[.044, .054]$ \\
\hline 5. Four-correlated factor ESEM & $171.710^{*}$ & 62 & .968 & .919 & .020 & $.038[.031, .045]$ \\
\hline 6. Four-correlated factor CFA - calibration & $1001.145^{*}$ & 203 & .934 & .911 & .050 & $.049[.044, .054]$ \\
\hline 7. Four-correlated factor CFA - validation & $994.326^{*}$ & 203 & .944 & .909 & .052 & $.051[.049, .055]$ \\
\hline 6. Four-correlated factor CFA - female & $889.352 *$ & 203 & .916 & .901 & .052 & $.049[.041, .058]$ \\
\hline 7. Four-correlated factor CFA - male & $894.658 *$ & 203 & .912 & .902 & .057 & $.065[.058, .072]$ \\
\hline 8. CFA $\rightarrow$ PLCQP & $741.021 *$ & 160 & .912 & .903 & .073 & $.059[.051, .069]$ \\
\hline
\end{tabular}

Note: PLCP = Perceived Locus of Causality Questionnaire Portuguese version; $\chi 2$ = chi-square test; $\mathrm{df}=$ degrees of freedom; CFI = Comparative Fit Index; TLI = Tucker-Lewis Index; SRMR = Standardized Root Mean Square Residual; RMSEA = Root Mean Squared Error of Approximation; 90\% $\mathrm{CI}=90 \%$ Confidence Interval of RSMEA; $* p<0.001$

well as other studies using this instrument (Cervelló-Gimeno et al. 2010).

According to current results, items loaded their pre-defined factor significantly. However, some items in the four-correlated factor CFA (item 5, 12, and 22) and several items in the fourcorrelated factor ESEM model (items 5, 7, 11, 19, 20, 21, and 22) displayed factor loadings below the cutoff of 0.50 (Hair et al. 2019). In line with several authors (Byrne 2011; Kline 2016), factor loadings below acceptable scores should be removed. But as stated by Hair et al. (2019), this is only a guideline and analyses should move forward if items loaded significantly their respective factor. Current findings showed that all items significantly loaded their predefined factor, in both fourcorrelated factor CFA and ESEM models and no significant cross-loadings were detected. The removal of items with loadings of $<0.50$ did not increased model fit, as the model tended to provide poorer fit to the data. Additionally, present results confirmed that all dimensions were internally consistent, as values of composite reliability were above cutoffs in both CFA and ESEM model specifications (Hair et al. 2019; Raykov 1997). Thus, current results support the reliability and validity of the measure (Hair et al. 2019).

The AVE scores values were above 0.50 indicating convergent validity. Hence, it is possible to confirm that items regarding learning and performance-oriented climates by teachers and peers do function as intended. As stated previously, all factorial weights were significant in their respective factors with no detected cross-loadings, suggesting good convergent validity (Byrne 2011). We examined and achieved discriminant validity for all possible comparisons supporting the distinctness of learning and performance climates oriented by teachers and peers. These results suggest that all four factors inherent in this translated and validated LAPOPECQ Portuguese version are indeed distinguishable (Hair et al. 2019), supporting empirical results (Papaioannou 1994, 1998).

\section{Multigroup Analysis}

With respect to the measurement invariance analysis between our two samples and gender, the four-correlated factor CFA model was equivalent across groups since invariance assumptions were respected between configural model and the others (i.e., metric, scalar, and residual). Regardless of samples or gender groups, the measurement model of the translated LAPOPECQ showed: the same set of items between groups (configural invariance); factor structure and factor loadings were equal between groups (weak factorial invariance); item factor structure, factor loadings, and item thresholds were equal between groups (strong invariance); and, item factor structure, factor loadings, item thresholds, and item residuals were equal between groups (strict factorial invariance).

Our results support equivalence of the LAPOPECQ between female and male students as reported previously (White and Duda 1994). However, other studies have reported differences in orientations between gender (Flores et al. 2008). Results may be explained through the importance of selfefficacy and internalization of the behavior, since boys may receive more acknowledgements for winning and outperforming other classmates than girls (Flores et al. 2008). However, to the best of our knowledge, this was the first attempt of conducting multigroup analysis between gender using a four-correlated factor CFA and ESEM models. Thus, further evidence of equivalence of for the LAPOPECQ between gender in other cultures seems of upmost importance.

\section{Nomological Validity}

Data from the bivariate correlation analysis indicated that learning-oriented climates are positively correlated with autonomous motivation and negatively associated with controlled motivation. These results are similar to the ones 
Table 3 Factor loadings of the four-correlated factors CFA and ESEM models

\begin{tabular}{|c|c|c|c|c|c|}
\hline & \multirow{2}{*}{$\begin{array}{l}\text { CFA } \\
\lambda\end{array}$} & \multicolumn{4}{|l|}{ ESEM } \\
\hline & & LCT $\lambda$ & PCT $\lambda$ & $\operatorname{LCS} \lambda$ & $\operatorname{PCS} \lambda$ \\
\hline Learning-oriented climate by PE Teacher (LCT) & .70 & .71 & & & \\
\hline Item 1 & $.58 * *$ & $.61^{* *}$ & .00 & -.02 & .05 \\
\hline Item 3 & $.59 * *$ & $.60 * *$ & -.06 & .09 & .05 \\
\hline Item 5 & $.46 * *$ & $.45^{* *}$ & -.07 & .11 & .02 \\
\hline Item 7 & $.50 * *$ & $.47 * *$ & $-.10 *$ & $.13^{*}$ & .03 \\
\hline Item 9 & $.56 * *$ & $.55 * *$ & -.03 & .03 & -.02 \\
\hline Item 11 & $.49 * *$ & $.41 * *$ & $.26 * *$ & .04 & $-.14 * *$ \\
\hline Performance-oriented climate by PE Teacher (PCT) & .78 & & .77 & & \\
\hline Item 2 & $.60 * *$ & $-.28 * *$ & $.52 * *$ & .09 & .07 \\
\hline Item 4 & $.55 * *$ & .12 & $.55 * *$ & -.05 & $.17 * *$ \\
\hline Item 6 & $.76 * *$ & -.08 & $.71 * *$ & -.03 & .01 \\
\hline Item 8 & $.63 * *$ & -.03 & $.63 * *$ & -.03 & -.03 \\
\hline Item 10 & $.64 * *$ & $.18^{*}$ & $.69 * *$ & -.06 & .05 \\
\hline Item 12 & $.47 * *$ & -.04 & $.50 * *$ & .07 & -.03 \\
\hline Learning-oriented climate by peers (LCS) & .75 & & & .73 & \\
\hline Item 13 & $.64 * *$ & -.02 & $.10^{*}$ & $.73 * *$ & -.09 \\
\hline Item 15 & $.69 * *$ & -.04 & .01 & $.75 * *$ & -.02 \\
\hline Item 17 & $.54 * *$ & .00 & -.05 & $.51 * *$ & .07 \\
\hline Item 19 & $.58 * *$ & .08 & -.08 & $.49 * *$ & .07 \\
\hline Item 21 & $.59 * *$ & $.19^{*}$ & -.02 & $.43 * *$ & .04 \\
\hline Performance-oriented climate by peers (PCS) & .73 & & & & .71 \\
\hline Item 14 & $.66^{* *}$ & .00 & -.06 & .03 & $.70 * *$ \\
\hline Item 16 & $.63 * *$ & -.05 & $.10^{*}$ & .06 & $.59 * *$ \\
\hline Item 18 & $.69 * *$ & .05 & -.02 & -.01 & $.70 * *$ \\
\hline Item 20 & $.50 * *$ & -.04 & $.11 *$ & -.07 & $.48 * *$ \\
\hline Item 22 & $.45 * *$ & .04 & .09 & .09 & $.39 * *$ \\
\hline
\end{tabular}

Note: $\lambda=$ factor loadings; target loadings are in bold; composite reliability coefficients are in italic; $* p<0.05 ; * * \mathrm{p}<0.001$

reported by (Cid et al. 2019; Moreno-Murcia et al. 2018) showing that learning-oriented climates perceived by students are related to positive outcomes such as autonomous motivation, sport participation, and/or intentions to practice extracurricular sport. On the other hand, performanceoriented climates were positively and significantly correlated
Table 4 Multigroup analysis of the four-correlated CFA measurement model across groups

\begin{tabular}{lllllllll}
\hline & \multirow{2}{*}{$\chi^{2}$} & $\Delta \chi^{2}$ & $\mathrm{df}$ & $\Delta \mathrm{df}$ & $\mathrm{CFI}$ & $\Delta \mathrm{CFI}$ & $\mathrm{TLI}$ & $\Delta$ TLI \\
\hline Calibration - Validation & & & & & & & & \\
$\quad$ Configural Invariance & $641.447^{*}$ & - & 198 & - & .910 & - & .905 & - \\
Weak Factorial Invariance & $652.635^{*}$ & 11.188 & 208 & 10 & .909 & .000 & .904 & .001 \\
Strong Invariance & $695.141^{*}$ & 53.694 & 220 & 12 & .909 & .000 & .901 & .004 \\
$\quad$ Strict Factorial Invariance & $710.325^{*}$ & 68.878 & 224 & 12 & .907 & .003 & .900 & .005 \\
Female - Male & & & & & & & & \\
Configural Invariance & $597.487^{*}$ & - & 198 & - & .912 & - & .902 & - \\
Weak Factorial Invariance & $616.973^{*}$ & 19.486 & 208 & 10 & .910 & .002 & .900 & .002 \\
Strong Invariance & $650.229^{*}$ & 52.742 & 220 & 12 & .908 & .002 & .899 & .003 \\
Strict Factorial Invariance & $684.204^{*}$ & 86.717 & 224 & 12 & .906 & .004 & .899 & .003 \\
\hline
\end{tabular}

Note: $\chi^{2}=$ chi-square; $\Delta \chi 2=$ difference in $\chi 2 ; \mathrm{df}=$ degrees of freedom; $\Delta \mathrm{df}=$ differences in $\mathrm{df} ; \mathrm{CFI}=$ Comparative Fit Index; $\Delta \mathrm{CFI}=$ differences in CFI; TLI = Tucker-Lewis Index; $\Delta \mathrm{TLI}=$ differences in TLI; * $p<0.001$ 
with controlled motivation and negatively with autonomous motivation. These results corroborate with previous studies (Flores et al. 2008; Moreno-Murcia et al. 2018; Papaioannou 1998), in which they have found that perception of a performance-oriented class was positively related to negative outcomes (i.e., controlled motivation, burnout, lack of interest). Results from the present study support the associations of motivational climates and behavioral regulations and are consistent with past literature (Flores et al. 2008; Warburton 2017).

According to results, performance-oriented climate by peers was positively correlated with autonomous motivation. Peers' goal normative and self-referenced criteria could be positively related to how they regulate their behaviors towards PE classes. As stated by Duda and Ntoumanis (2003), perceiving performance-oriented climates is not evidently problematic. For example, students who are very confident and skillful can correspond to high performance both at a physical and psychological level, thus, influencing other to expand their capacities through more self-determined motivation.

\section{Conclusion}

In sum, current results proved support for the construct validity of the LAPOPECQ adding new evidence for the construct distinctiveness of learning and performance-oriented climates by teachers and peers in a sample of Portuguese students. The present study reinforces the important of measuring students' perception of motivational climates at the situational level, since motivational climates induced PE teachers and peers are differently associated with behavioral regulations towards physical education.

PE teachers should use the LAPOPECQ during PE classes to assess students' perception of learning and performanceoriented climates as a way to understand how students perceive PE teacher class conduct and peer interactions. In addition, assessing motivational climates is crucial, since learning and performance-oriented climates can have an effect on how students will regulate their behaviors towards physical education classes (Cid et al. 2019). Measuring students' perceptions of motivational climates in advance could give PE teachers the necessary tools to create and adapt PE classes so students would engage more actively in PE classes and experience positive outcomes and achieve higher academic success.

\section{Limitations and Agenda for Future Research}

Despite our study being based on strong and robust theoretical framework, the present study had some limitations. First, this was the first attempt to translate and validate the LAPOPECQ in a sample of Portuguese students. Therefore, our findings cannot be generalized to other cultures. Although the
LAPOPECQ has been validated in different cultures such as Greek (Papaioannou 1998) and Spanish (Cervelló-Gimeno et al. 2010), more studies are warranted to establish cultural equivalence. Nevertheless, the four-correlated factor model specifications provided acceptable fit to the data showing similar results reported by previous studies (Cervelló-Gimeno et al. 2010; Papaioannou 1994).

Second, even though results displayed robust associations between motivational climates and behavioral regulations, there are other cognitive and emotional that could emerge as consequences. For instance, Cid et al. (2019) have shown learning-oriented climates to be associated with basic psychological needs satisfaction and intentions to maintain leisuretime sport participation. Other (Moreno-Murcia et al. 2018; Moreno-Murcia et al. 2011) reported performance-oriented climates to predict controlled motivation and lower levels of satisfaction with life. Thus, forthcoming studies should examine other interactions between motivational climates and emotional and cognitive consequences in Portuguese students.

Acknowledgments This work was supported by the National Funding through the Portuguese Foundation for Science and Technology, I.P., under the project UIDP/04748/2020 for the author Filipe Rodrigues; and under the project UID04045/2020 for the authors Diogo Monteiro and Luís Cid.

\section{Compliance with Ethical Standards}

Conflict of Interest No conflict to declare.

\section{References}

Allison, P. (2000). Multiple imputation for missing data: A cautionary tale. Sociological Methods \& Research, 28(3), 301-309. https://doi. org/10.1177/0049124100028003003.

Ames, C. (1992). Achievement goals, motivational climate and motivational processes. In G. Roberts (Ed.), Motivation in sport and exercise (pp. 161-176). Champaign: Human Kinetics.

Ames, C., \& Archer, J. (1988). Achievement goals in the classroom: Students' learning strategies and motivation processes. Journal of Educational Psychology, 80(3), 260-267. https://doi.org/10.1037/ 0022-0663.80.3.260.

Bortoli, L., Bertollo, M., Filho, E., di Fronso, S., \& Robazza, C. (2017). Implementing the TARGET model in physical education: Effects on perceived Psychobiosocial and motivational states in girls. Frontiers in Psychology, 8, 1517. https://doi.org/10.3389/fpsyg.2017.01517.

Brislin, R. (1970). Back-translation for cross-cultural research. Journal of Cross-Cultural Psychology, 1(3), 187-216. https://doi.org/10.1177/ 135910457000100301.

Brislin, R. (1980). Translation and content analysis of oral and written material. In H. C. Triandis \& R. W. Brislin (Eds.), Handbook of cross-cultural psychology (pp. 389-444). Boston: Allyn and Bacon.

Browne, M. (2001). An overview of analytic rotation in exploratory factor analysis. Multivariate Behavioral Research, 36(1), 111-150.

Byrne, B. (2011). Structural Equation Modeling with Mplus - Basic Concepts, Applications, and Programming: Routledge.

Cervelló-Gimeno, E. M., Jiménez-Castuera, R., Moya-Ramón, M., \& Moreno-Murcia, J. A. (2010). Validation of the Spanish language 
version of the learning and performance orientations in physical education classes questionnaire. (Validación de la versión Española del Cuestionario de Orientaciones al Aprendizaje y el Rendimiento en las clases de Educación Física). RICYDE. Revista Internacional de Ciencias del Deporte, 6(20), 242-253. https://doi. org/10.5232/ricyde2010.02006.

Chen, F. (2007). Sensitivity of goodness of fit indexes to lack of measurement invariance. Structural Equation Modeling: A Multidisciplinary Journal, 14(3), 464-504. https://doi.org/10.1080/ 10705510701301834.

Chen, F. (2008). What happens if we compare chopsticks with forks? The impact of making inappropriate comparisons in cross-cultural research. Journal of Personality \& Social Psycholgy, 95(5), 10051018. https://doi.org/10.1037/a0013193.

Cheung, G., \& Rensvold, R. (2002). Evaluating goodness-of-fit indexes for testing measurement invariance. Structural Equation Modeling: A Multidisciplinary Journal, 9(2), 233-255. https://doi.org/10.1207/ s15328007sem0902 5.

Cid, L., Pires, A., Borrego, C., Mendes, P., Teixeira, D., Moutao, J., \& Monteiro, D. (2019). Motivational determinants of physical education grades and the intention to practice sport in the future. PLoS One, 14(5), e 0217218. https://doi.org/10.1371/journal.pone.0217218.

Duda, J. (2013). The conceptual and empirical foundations of empowering coaching ${ }^{\mathrm{TM}}$ : Setting the stage for the PAPA project. International Journal of Sport and Exercise Psychology, 11, 311(4), -318. https://doi.org/10.1080/1612197x.2013.839414.

Duda, J. L., \& Ntoumanis, N. (2003). Correlates of achievement goal orientations in physical education. International Journal of Educational Research, 39(4-5), 415-436. https://doi.org/10.1016/ j.ijer.2004.06.007.

Fernandes, H., \& Vasconcelos-Raposo, J. (2005). Self-determination continuum: Validity for its application in the sports context. Psychology Studies (Natal), 10(3), 385-395. https://doi.org/10. 1590/S1413-294X2005000300007.

Flores, J., Salguero, A., \& Márquez, S. (2008). Goal orientations and perceptions of the motivational climate in physical education classes among Colombian students. Teaching and Teacher Education, 24(6), 1441-1449. https://doi.org/10.1016/j.tate.2007.11.006.

Gravetter, F., \& Wallnau, L. (2014). Essentials of statistics for the behavioral sciences (8th ed.). Cengage Learning: Wadsworth, Belmont, CA.

Hair, J., Black, W., Babin, B., \& Anderson, R. (2019). Multivariate Data Analysis (8th ed.): New Jersey: Pearson educational, Inc.

Huéscar Hernández, E., Moreno-Murcia, J. A., Cid, L., Monteiro, D., \& Rodrigues, F. (2020). Passion or perserverance? The effect of perceived autonomy support and grit on academic performance in college students. International Journal of Environmental Research and Public Health, 17, 2143. https://doi.org/10.3390/ijerph17062143.

Kline, R. (2016). Principles and practice of structural equation modelling (3rd ed.): New York: The Guildford press.

Marsh, H., Guo, J., Dicke, T., Parker, P., \& Craven, R. (2019). Confirmatory factor analysis (CFA), exploratory structural equation modeling (ESEM), and set-ESEM: Optimal balance between goodness of fit and parsimony. Multivariate Behavioral Research, 55, 118. https://doi.org/10.1080/00273171.2019.1602503.

Marsh, H., Hau, K., \& Wen, Z. (2004). In search of Golden rules: Comment on hypothesis-testing approaches to setting cutoff values for fit indexes and dangers in overgeneralizing $\mathrm{Hu}$ and Bentler's (1999) findings. Structural Equation Modeling: A Multidisciplinary Journal, 11(3), 320-341. https://doi.org/10.1207/ s15328007sem1103 2.

Moreno-Murcia, J., Huescar Hernandez, E., \& Ruiz, L. (2018). Perceptions of controlling teaching behaviors and the effects on the motivation and behavior of high school physical education students. International Journal of Environmental Ressearch \& Public Health, 15(10). https://doi.org/10.3390/ijerph15102288.
Moreno-Murcia, J., Sicilia, Á., Cervelló, D., Huescar Hernandez, E., \& Dumitru, D. (2011). The relationship between goal orientations, motivational climate and Selfreported discipline in physical education. Journal of Sports Science \& Medicine, 10(1), 119-129.

Morin, A., Arens, A., \& Marsh, H. (2015). A Bifactor exploratory structural equation modeling framework for the identification of distinct sources of construct-relevant psychometric multidimensionality. Structural Equation Modeling: A Multidisciplinary Journal, 23(1), 116-139. https://doi.org/10.1080/10705511.2014.961800.

Muthén, L., \& Muthén, B. (2010). Mplus User's Guide: Muthén \& Muthén: Los Angeles.

Nicholls, J. (1984). Achievement motivation: Conceptions of ability, subjective experience, task choice, and performance. Psychological Review, 91(3), 328-346. https://doi.org/10.1037/0033-295X.91.3.328.

Ntoumanis, N., \& Biddle, S. (1999). A review of motivational climate in physical activity. Journal of Sports Sciences, 17(8), 643-665. https://doi.org/10.1080/026404199365678.

Papaioannou, A. (1994). Development of a questionnaire to measure achievement orientations in physical education. Research Quarterly for Sport and Exercise, 65(1), 11-20. https://doi.org/10. 1080/02701367.1994.10762203.

Papaioannou, A. (1998). Students' perceptions of the physical education class environment for boys and girls and the perceived motivational climate. Research Quarterly for Sport and Exercise, 69(3), 267275. https://doi.org/10.1080/02701367.1998.10607693.

Pineda-Espejel, H., Lopez-Walle, J., \& Tomas, I. (2017). Situational and dispositional factors that predict motivation: A multilevel study. Spanish Journal of Psychology, 20, 1-20. https://doi.org/10.1017/ sjp.2017.17.

Raykov, T. (1997). Estimation of composite reliability for congeneric measures. Applied Psychological Measurement, 21(2), 173-184. https://doi.org/10.1177/01466216970212006.

Ryan, R. (1995). Psychological needs and the facilitation of integrative processes. Journal of Personality, 63(3), 397-427. https://doi.org/ 10.1111/j.1467-6494.1995.tb00501.x.

Ryan, R., \& Deci, E. (2017). Self-determination theory. Basic Psychological Needs in Motivation, Development, and Wellness: New York: Guildford Press.

Ryan, R., \& Deci, E. (2019). Brick by brick: The origins, development, and future of self determination theory. Advances in Motivation Science, 6, 111-156. https://doi.org/10.1016/bs.adms.2019.01.001.

Standage, M., Gillison, F., \& Treasure, D. (2007). Self-determination and motivation in physical education. In M. Hagger \& N. Chatzisarantis (Eds.), Intrinsic motivation and self-determination in exercise and sport. Champaign: Human Kinetics.

Sass, D. (2011). Testing measurement invariance and comparing latent factor means within a confirmatory factor analysis framework. Journal of Psychoeducational Assessment, 29(4), 347-363. https:// doi.org/10.1177/0734282911406661.

Vasconcellos, D., Parker, P., Hilland, T., Cinelli, R., Owen, K., Kapsal, N., Lee, J., Antczak, D., Ntoumanis, N., Ryan, R., \& Lonsdale, C. (2019). Self-determination theory applied to physical education: A systematic review and meta-analysis. Journal of Educational Psychology. https://doi.org/10.1037/edu0000420.

Warburton, V. (2017). Peer and teacher influences on the motivational climate in physical education: A longitudinal perspective on achievement goal adoption. Contemporary Educational Psychology, 51, 303-314. https://doi.org/10.1016/j.cedpsych.2017. 08.001 .

White, S. A., \& Duda, J. L. (1994). The relationship of gender, level of sport involvement, and participation motivation to task and ego orientation. International Journal of Sport Psychology, 25(1), 4-18.

Publisher's Note Springer Nature remains neutral with regard to jurisdictional claims in published maps and institutional affiliations. 